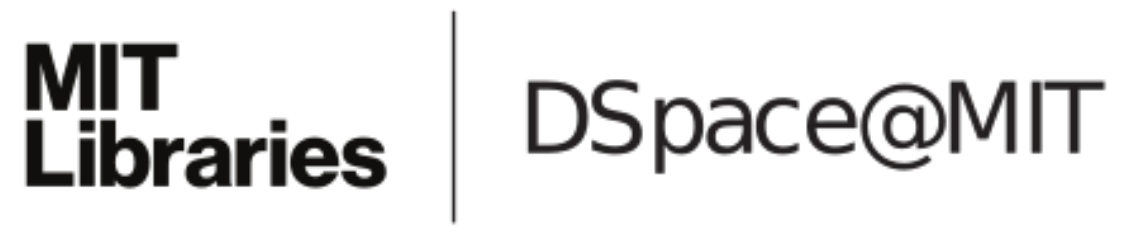

\author{
MIT Open Access Articles
}

Using the variogram for vector outlier screening: application to feature-based image registration

The MIT Faculty has made this article openly available. Please share how this access benefits you. Your story matters.

Citation: Luo, Jie et al. "Using the variogram for vector outlier screening: application to featurebased image registration." International Journal of Computer Assisted Radiology and Surgery 13 (2018): 1871-1880 @ 2018 The Author(s)

As Published: $10.1007 / \mathrm{S11548-018-1840-5}$

Publisher: Springer Nature

Persistent URL: https://hdl.handle.net/1721.1/125384

Version: Author's final manuscript: final author's manuscript post peer review, without publisher's formatting or copy editing

Terms of use: Creative Commons Attribution-Noncommercial-Share Alike 


\title{
Using the variogram for vector outlier screening: application to feature-based image registration
}

\author{
Jie Luo ${ }^{1,2}$, Sarah Frisken ${ }^{1}$, Ines Machado ${ }^{1}$, Miaomiao Zhang ${ }^{3}$, Steve Pieper ${ }^{1}$, Polina \\ Golland $^{4}$, Matthew Toews ${ }^{5}$, Prashin Unadkat ${ }^{1}$, Alireza Sedghi ${ }^{1}$, Haoyin Zhou ${ }^{1}$, Alireza \\ Mehrtash ${ }^{1}$, Frank Preiswerk ${ }^{1}$, Cheng-Chieh Cheng ${ }^{1}$, Alexandra Golby ${ }^{1}$, Masashi \\ Sugiyama $^{6,2}$, and William M. Wells III $^{1,4}$ \\ 'Brigham and Women's Hospital, Harvard Medical School, 75 Francis St., Boston, MA 02115, \\ USA \\ ${ }^{2}$ Graduate School of Frontier Sciences, The University of Tokyo, 5 Chome-1-5 Kashiwanoha, \\ Kashiwa, Chiba 277-8561, Japan \\ ${ }^{3}$ Computer Science and Engineering Department, Lehigh University, 19 Memorial Drive West, \\ Bethlehem, PA 18015, USA \\ ${ }^{4}$ Computer Science and Artificial Intelligence Laboratory, Massachusetts Institute of Technology, \\ 32 Vassar St, Cambridge, MA 02139, USA \\ ${ }^{5}$ Ecole de Technologie Superieure, 1100 Rue Notre-Dame Ouest, Montreal, H3C 1K3, Canada \\ ${ }^{6}$ Center for Advanced Intelligence Project, RIKEN, 1-4-1 Nihonbashi, Chuo-ku, 103-0027, Tokyo, \\ Japan
}

\section{Abstract}

Purpose: Matching points that are derived from features or landmarks in image data is a key step in some medical imaging applications. Since most robust point matching algorithms claim to be able to deal with outliers, users may place high confidence in the matching result and use it without further examination. However, for tasks such as feature-based registration in image-guided neurosurgery, even a few mismatches, in the form of invalid displacement vectors, could cause serious consequences. As a result, having an effective tool by which operators can manually screen all matches for outliers could substantially benefit the outcome of those applications.

Methods: We introduce a novel variogram-based outlier screening method for vectors. The variogram is a powerful geostatistical tool for characterizing the spatial dependence of stochastic processes. Since the spatial correlation of invalid displacement vectors, which are considered as

\footnotetext{
jluo5@bwh.harvard.edu.

${ }^{6}$ Statements

Conflicts of interests: The authors declare that they have no conflict of interest.

Ethical approval: All procedures performed in studies involving human participants were in accordance with the ethical standards of the institutional and/or national research committee and with the 1964 Helsinki declaration and its later amendments or comparable ethical standards.

Informed consent: Informed consent was obtained from all individual participants included in the study.
} 
vector outliers, tends to behave differently than normal displacement vectors, they can be efficiently identified on the variogram.

Results: We validate the proposed method on 9 sets of clinically acquired ultrasound data. In the experiment, potential outliers are flagged on the variogram by one operator, and further evaluated by 8 experienced medical imaging researchers. The matching quality of those potential outliers are approximately 1.5 lower, on a scale from 1 (bad) to 5 (good), than valid displacement vectors.

Conclusion: The variogram is a simple yet informative tool. While being used extensively in geostatistical analysis, it has not received enough attention in the medical imaging field. We believe there is a good deal of potential for clinically applying the proposed outlier screening method. By way of this paper, we also expect researchers to find variogram useful in other medical applications that involve motion vectors analyses.

\section{Keywords}

Vector Outlier Screening; Variogram; Feature-Based Registration; Neurosurgery

\section{Introduction}

Matching points that are derived from features or landmarks in image data is a key step in many medical imaging applications, such as 3D reconstruction and image registration for surgical navigation. Since most robust point matching algorithms claim to be able to deal with outliers [1-5], users may place high confidence in the matching result and use it without further examination. However, for tasks where precision is of paramount importance, even a few mismatches can cause serious consequences. As a result, having an effective tool by which operators can manually screen all matches for outliers could substantially benefit the outcome of those applications.

Image-guided neurosurgery is a task that requires precise localization of tumor boundaries [6]. Conventionally, surgeons use commercial Image Guided Neuro-navigation Systems (IGNSs) to map the preoperative image data to an intraoperative patient coordinate system so as to get an updated view of the brain [7, 8]. Unfortunately, intraoperative brain deformation, also known as brain shift, invalidates this mapping and limits the trustworthiness of using preoperative images in intraoperative surgical navigation [9, 10]. In order to compensate the brain shift and increase the accuracy of neurosurgery, non-rigid image registration [11-14] is sometimes adopted to spatially align the preoperative and intraoperative images.

Because of tumor resection, the same structure may not exist on both preoprative and intraoperative images, hence Feature-Based Registration (FBR) becomes a promising strategy due to its robustness in registering images with missing correspondence [15]. FBR consists of three steps. In the feature extraction step, distinctive local image features are automatically extracted and identified as key-points on preoperative and intraoperative images. In the feature matching step, a matcher searches for a corresponding intraoperative key-point for each key-point on the preoperative image. From every matched key-point pair, a displacement vector can be obtained to indicate the movement of the preoperative key- 
point due to the brain shift process. In the last step of FBR, the algorithm generates a dense deformation field for the entire preoperative image from the displacement vectors and uses it to map the preoperative image to the intraoperative space. If invalid displacement vectors, which falsely reflect the tissue movement under brain shift, are obtained from mismatched key-point pairs, it will negatively affect the registration result.

In this paper, we introduce a simple yet informative variogram-based outlier screening method for vectors and demonstrate its usefulness in the context of feature-based registration. While variograms are extensively used in geostatistics to capture the spatial dependence of stochastic processes [16], they have not yet received much attention in the medical imaging field. Since the spatial correlation of invalid displacement vectors, which are considered vector outliers, tend to behave differently than valid displacement vectors, they can be identified on the variogram cloud and flagged for further examinations. The paper is organized as follows: In Section 2, we explain how to estimate and understand the variogram in the FBR context. In Section 3, we demonstrate how to identify outliers on the variogram. Finally, experimental results and conclusion are presented in Section 4.

\section{Summarizing variogram in FBR}

The variogram is a powerful tool that is originated in geostatistics. In this section, we summarize the variogram in the context of FBR.

In the feature matching step of FBR, we obtain a displacement vector from every matched key-point pair. For example, as shown in Fig. 1(a)(b), assume $\mathbf{k}_{p}$ is the coordinate of an extracted preoperative key-point, and $\mathbf{k}_{i}$ is the coordinate of its corresponding intraoperative counterpart. After overlaying both images in Fig.1(c), the displacement vector $\mathbf{d}$ from the preoperative key-point pointing to the intraoperative key-point can be calculated as $\mathbf{k}_{i}-\mathbf{k}_{p}$.

All displacement vectors form a vector field, which is supposed to indicate the intraoperative brain shift. We attempt to model the spatial dependence of the vector field and use the dependency as a criterion for identifying displacement vector outliers.

\subsection{Random process model}

Brain shift is caused by the interaction of physical, surgical and biological factors. Due to the complexity and incomplete understanding of the phenomenon, we model the deformation of the brain as a stochastic process. In our model, let $\mathbf{x} \in \mathbb{R}^{d}$ be a generic data location in the $d$-dimensional Euclidean space.

Suppose $\mathbf{Z}(\mathbf{x})$ is a random quantity at location $\mathbf{x}$, its realization $\mathbf{z}(\mathbf{x})$ is a displacement vector like the one in Fig.1(d). Given an index set $D \subset \mathbb{R}^{d}$, we can model the vector field as

$$
\{\mathbf{Z}(\mathbf{x}): \mathbf{x} \in D\}
$$

For brain shift, the deformation at a particular location is likely to have influence in all directions. Capturing the spatial correlation of deformation is essential for understanding the 
phenomenon. The definition of the variogram comes naturally as we quantify the spatial dependence of $\mathbf{Z}(\mathbf{x})$.

\subsection{The variogram and empirical variogram}

The pairwise dependence between displacement vectors in a random field is the variance of the difference between the values at two locations across the field [16]:

$$
2 \gamma\left(\mathbf{x}_{1}, \mathbf{x}_{2}\right):=\operatorname{var}\left(\mathbf{Z}\left(\mathbf{x}_{1}\right)-\mathbf{Z}\left(\mathbf{x}_{2}\right)\right)
$$

We make the mild assumption that the random field has a constant mean and is intrinsically stationary. Intrinsic stationarity states that the variance of difference is the same between any two points that are separated by the same distance. Adopting a terminology from time series analysis, we represent the separation between two spatial locations by a lag vector $\mathbf{h}=\mathbf{x}_{2}$ $\mathbf{x}_{1}$ and rewrite (2) as

$$
\begin{aligned}
2 \gamma\left(\mathbf{x}_{1}, \mathbf{x}_{2}\right) & =E\left[\left(\left(\mathbf{Z}\left(\mathbf{x}_{1}\right)-\mathbf{Z}\left(\mathbf{x}_{1}+\mathbf{h}\right)\right)^{2}\right]\right. \\
& =2 \gamma(h) .
\end{aligned}
$$

$\mathcal{Y}(h)$, also known as the variogram (sometimes is called the semivariogram) of a random field $\mathbf{Z}(\mathbf{x})$, is defined as one-half the average squared difference between the value at locations separated by distance $h$ [17]. The variogram function $\gamma(h)$ depends only on $h$ and it measures the correlation as a function of distance.

Notice that $\gamma(h)$ is a theoretical function. In practice, we do not have the displacement vector for every location to estimate $\chi(h)$, so we use its empirical alternative [18]. This definition will be clarified in Section 2.3.

$$
\widehat{\gamma}(h \pm \delta):=\frac{1}{2|N(h \pm \delta)|} \sum_{(i, j) \in N(h \pm \delta)}\left\|\mathbf{z}\left(\mathbf{x}_{\mathbf{i}}\right)-\mathbf{z}\left(\mathbf{x}_{j}\right)\right\|^{2}
$$

\subsection{Estimating the empirical variogram in FBR}

In FBR, there are several ways to represent $\left\|\mathbf{z}\left(\mathbf{x}_{i}\right)-\mathbf{z}\left(\mathbf{x}_{j}\right)\right\|$ and $h$. In this paper, we use the following representations. In Fig.2(a), on a preoperative Magnetic Resonance Image (MRI), there are two key-points whose coordinate are $\mathbf{x}_{1}$ and $\mathbf{x}_{2} . \mathbf{z}\left(\mathbf{x}_{1}\right)$ and $\mathbf{z}\left(\mathbf{x}_{2}\right)$ are their associated displacement vectors. As can be seen in Fig.2(b), $\left\|\mathbf{z}\left(\mathbf{x}_{1}\right)-\mathbf{z}\left(\mathbf{x}_{2}\right)\right\|$ is equal to the norm of the vector $\mathbf{z}\left(\mathbf{x}_{2}\right)-\mathbf{z}\left(\mathbf{x}_{1}\right)$, and lag $h$ is the Euclidean distance between the two key-points $\| \mathbf{x}_{2}-$ $\mathbf{x}_{1} \|$.

Estimating an empirical variogram can be analogous to building a histogram based on samples from a continuous distribution. The strategy is to group pairs of points that have similar $h$ into the same bin, and approximate the variogram by combining information from 
all of the bins. Here we illustrate the 4 steps of estimating an empirical variogram using Fig. 3.

(a) Construct the variogram cloud by plotting $\left\|\mathbf{z}\left(\mathbf{x}_{i}\right)-\mathbf{z}\left(\mathbf{x}_{j}\right)\right\|^{2}$ for all key-point pairs with their lag $h_{i j}$ :

(b) Introduce a variable $\delta$ as the tolerance range for lag $h$, and divide the variogram cloud into bins whose width is set to $2 \delta$.

(c) Calculate the mean $\hat{\gamma}\left(h_{\text {bink }} \pm \delta\right)$, which are marked as blue in Fig.3(c), for every bin using Equation (4). $\left|N\left(h_{\text {bink }} \pm \delta\right)\right|$ represents the number of key-point pairs of bin $k$.

(d) Plotting all $\hat{\gamma}\left(h_{\mathrm{bink}} \pm \delta\right)$ with their $h_{\mathrm{bin} i}$ to obtain the empirical variogram.

The lag tolerance $\delta$ should be neither too large nor too small, since it may veil the shortdistance correlations or produce empty bins. Typically, $\delta$ is determined in Ad hoc fashion. When $\delta=0$, the empirical variogram $\hat{\gamma}(h \pm \delta)$ is an unbiased estimator of the theoretical variogram $\mathcal{\gamma}(h)$ [16]. Notice that the proposed variogram based outlier screening method primarily uses the variogram cloud, hence the choice of $\delta$ has no impact on its performance.

In Fig.4, we display a variogram cloud and the empirical variogram estimated from a set of preoperative and intraoperative ulstrasound (US) data. For this US data, we were able to find 71 pairs of matched key-points, and the $\delta$ used to estimate the empirical variogram is $0.5 \mathrm{~mm}$. Here the $\mathrm{x}$-axis represent lag $h$, and the y-axis is the value difference among pairs. The empirical variogram does indicate that nearby key-points tend to have more similar displacement vectors than those that are far apart. In the rest of this article, we use the variogram and empirical variogram interchangeably.

\subsection{Understanding variograms}

In variogram-based diagnostics, it is often necessary to fit a model, that is a continuous function or curve, to the empirical variogram. Although methodology for fitting a model to the empirical variogram is beyond the scope of this paper, we use a fitted model to explain some important parameters of a variogram.

Fig.5(a) is an example of a fitted model. A fitted model is commonly described by the following characteristics:

Nugget When two key-points are extremely close, they should have very similar displacement vectors. In other words, at lag $h=0$, the variogram value should be 0 .

However, at a small separation distance, the variogram sometimes exhibits a nugget effect, which is some value greater than 0 .

Sill The value at which the model first flattens out.

Range It is the lag distance for which the sill is reached. Presumably, only samples that are separated by distances closer than the range are spatially correlated. 
In Fig.5(b), we fit a continuous model to the variogram in Fig.4(b). We can see insignificant nugget effect and an approximate $8.8 \mathrm{~mm}$ sill value. The range is around $21 \mathrm{~mm}$, which indicates that the spatial influence for a particular key-point to its neighbors is $21 \mathrm{~mm}$.

\section{Variogram and vector-outliers removal}

In FBR, the feature matching algorithm, e.g., [15], may identify feature correspondences between preoperative and intraoperative images solely based on the local image appearance similarity. While the algorithm is claimed to be robust against outliers, similar image patches and artefacts at non-corresponding locations of the brain will sometimes be extracted and matched. These mismatched key-point pairs produce invalid displacement vectors, as outliers, that fail to reflect the actual brain deformation. Since all displacement vectors will be used to generate the dense deformation field, we want a simple yet informative tool so that operators can efficiently screen all displacement vectors for outliers.

In Section 2, we mentioned that the variogram cloud can capture the spatial dependence of displacement vectors. Since the spatial correlation of vector outliers tend to behave differently than valid displacement vectors, they can be spotted on the variogram. There are two key features for the variogram-based outlier screening: 1) It is effective and easy to use. Operators can quickly spot and flag potential outliers on the variogram cloud. 2) The variogram is also very informative. Operators also can grasp the quality/trend of the vector field by observing variogram patterns.

\subsection{Global and local outliers in FBR}

Outliers are extreme values that deviate from other observations in the data. In FBR, there are generally two types of outliers [20]:

(1) A global outlier is a sample point that has a very high or a very low value compared to all the values in a dataset. For example, due to the symmetry of cerebral hemispheres, similar intensity patterns exist on the opposite half of the brain for MRI images. If these patterns are mismatched as corresponding keypoint pairs, they produce global outliers. These global outliers are clearly incorrect, and should be removed.

(2) A local outlier is a sample key-point that has a value within the normal range for the entire dataset, but is unusually high or low compared to its neighboring points. In FBR, some displacement vectors may seem to be local outliers at first, yet they capture the actual local deformations that are induced by the brain shift phenomenon. These key-points are vital for understanding and correcting the brain shift, and may be the most significant points in the registration. Therefore, it is unnecessary to remove all local outliers, but suppress them to a certain degree or perhaps to flag them for manual intervention.

\subsection{Outliers in the variogram cloud}

Here we explain how to look for global and local outliers in a variogram cloud by the following two examples. 
In a synthetic vector field shown in Fig.6(a), we have 25 valid displacement vectors and a manually added global outlier. Since the displacement vector of that global outlier is drastically different from other key-points, all pairings of points involving that global outlier, regardless of the lag distance $h$, have high $\left\|\mathbf{z}\left(\mathbf{x}_{i}\right)\right\| \mathbf{z}\left(\mathbf{x}_{j}\right) \|^{2}$ values. From the variogram cloud in Fig.6(b), we can see two main strata of points. All the higher values, which are marked as red, come from pairings with the manually added global outlier. On the other hand, the lower strata is composed of pairings among the rest of valid displacement vectors.

In another synthetic vector field shown in Fig.7(a), we have 25 valid displacement vectors and a manually added local outlier. The local outlier does not have high values across all distance in the variogram cloud. However, the variogram values of its close neighbors are relatively high. In Fig.7(b), all values from pairings with the local outlier are marked as red. On the bottom-left of the variogram cloud, we can notice a small cluster of abnormally high values as expected. Sometimes, even the local outlier is hard to notice in the displacement vector field, it is easy to spot in the variogram cloud.

Given the characteristics of global and local outliers, screening using the variogram is very straightforward. Since the variogram provides a distinct visualization for outliers, users can get an overview of potential outliers by simply looking at the patterns on the top and bottomleft corner of the variogram cloud. If abnormal variogram values are found, it is easy to trace back to the key-point matches for further examinations.

\section{Experiments}

In this section, we experimentally evaluate the proposed method.

\subsection{Outlier Screening}

In the experiments we tested the proposed variogram-based outlier screening method on a clinically acquired US data set. This 9-patient data set contains US images taken from 3 different stages of neurosurgery: before opening the dura (predura), after opening the dura (postdura) and during the tumor resection (intraoperative). We registered the intraoperative image to the predura image. In case the intraoperative image is not available, we register the postdura image instead.

After the feature matching, an operator quickly looked for abnormal patterns on the top and bottom-left corner of the variogram cloud and flagged the matches that are in correspondence with those patterns. All flagged matches, mixed with some valid matches, are further sent to 8 experienced medical imaging researchers for rating.

In the rating process, without knowing how many potential outliers are in the group, raters assign a score from 1 (bad) to 5 (good) to every match. After a zero-mean normalization for each rater, we calculated the score difference of every potential outlier to the mean score of valid matches and recorded it as Ourlier Score (OLS). The number of matches, number of potential outliers (\#OL) suggested by the variogram, and the OLS of each potential outlier are presented in table 1. Here OLS1 means the OLS for potential outlier 1, etc. 
The feature matching algorithm we use is claimed to be robust [15], yet despite the two cases which have less than 15 displacement vectors, we were able to flag at least one potential outlier using the variogram. Given the fact that all flagged potential outliers for cases 1-3 and cases 5-6 and 9 have substantially lower scores than normal matches, it is reasonable to conclude that adding an outlier screening step after the feature matching is beneficial.

Fig. 8 shows the variogram for all potential outliers that were flagged by the operator. In Fig. 9 , we show the axial, sagittal and coronal views of some found outlier matches together.

\subsection{Outlier suppression}

In case there are too many matched key-point pairs where a manual screening is too time consuming, we can combine the variogram with other statistical tools, such as Tukey's fences [21], to suppress outliers in a more systematic fashion.

The Inter Quartile Range (IQR) is the distance between the lower quartile $Q_{1}$ and the upper quartile $Q_{3}$. Tukey's fences can be stated as

$$
\left[Q_{1}-k \cdot \mathrm{IQR}, Q_{3}+k \cdot \mathrm{IQR}\right]
$$

Equation (5) is called an inner fence with $k=1.5$, and an outer fence with $k=3$.

Conventionally, a value between the inner and outer fences is a possible outlier. A value beyond the outer fence can be considered as an extreme outlier [21].

The FBR was mainly developed for registering US to US data. However, for US-US registration, the number of matched key-point pairs found is usually less than 100 . To demonstrate how to use the variogram and Tukey's fences to suppress outliers in massive vector fields, we used a pair of preoperative and intraoperative MRI images. After the feature matching, we found 386 matched key-point pairs, in which outliers are likely to present. Fig.10(a) and (b) show its variogram cloud and variogram respectively. The half-bin size used here is $\delta=1.5 \mathrm{~mm}$.

Based on the reasoning stated in Section 3, we use the inner fence to filter out all potential global outliers for bins whose $15 \mathrm{~mm}<h \leqq 80 \mathrm{~mm}$, and adopt the more conservative outer fence to suppress local outliers for $h \leqq 15 \mathrm{~mm}$.

In FBR, a standard way to measure the registration error is by the Sum of (Squared) Euclidean distance between all matched key-point pairs (SSD). The transformation matrix in affine registration is derived by minimizing the SSD in a least square fashion. In case there are bad matches, the algorithm, influenced by vector outliers, tends to obtain sub-optimal affine transformation matrix that leads to large SSD. In the preliminary experiment, after the outlier suppression, the SSD of feature-based affine registration dropped from $1.66 \mathrm{~mm}$ to $1.25 \mathrm{~mm}$, which we believe is a good indicator for having less outliers. 


\section{Conclusion}

Matching points that are derived from features or landmarks in image data is a key step in some medical application. For application that demand a high precision, such as featurebased preop-to-intraop registration, adding an outlier screening step could make it less prone to mismatches and improve the overall result. In this paper, we proposed to use the variogram to estimate the spatial dependence of displacement vectors. Since the spatial correlation of vector outliers behave differently than valid displacement vectors, they can be efficiently identified on the variogram and flagged for further examinations.

The variogram is a simple yet informative tool. While being used extensively in geostatistical analysis, the variogram has not received much attention in the medical imaging field. We believe there is a good deal of potential for clinically applying the variogram. For instance, with an interactive user interface, such as 3D Slicer [19], clinicians or their assistants can easily spot and flag outliers on the variogram for manual intervention. By way of this paper, we also expect researchers to find the variogram useful in other medical applications that involve motion vectors analyses.

\section{Acknowledgements}

This work was supported by National Institute of Health grants P41-EB015898-09 and P41-EB015902. This work was also supported by the International Research Center for Neurointelligence (WPI-IRCN) at The University of Tokyo Institute for Advanced Study.

\section{References}

1. Chui H, Rangarajan A (2003). A new point matching algorithm for non-rigid registration. Computer Vision and Image Understanding. Vol.89(2): 114141.

2. Fitzgibbon AW (2003). "Robust registration of 2D and 3D point sets". Image and Vision Computing. 21 (13): 11451153.

3. Fischler MA, Bolles RC (1981) Random sample consensus: a paradigm for model fitting with application to image analysis and automated cartography, Comm of the ACM, Vol.24 no.6, 381-395

4. Myronenko A, Song X (2009) Point set registration: coherent point drift, IEEE TPAMI, Vol.32(2), $2262-2275$

5. Jian B, Vemuri BC. (2011). Robust Point Set Registration Using Gaussian Mixture Models. IEEE Transactions on Pattern Analysis and Machine Intelligence. 33(8): 16331645.

6. Stummer W, Pichlmeier U, Meinel T, Wiestler OD, Zanella F, Reulen HJ (2006) Fluorescenceguided surgery with 5- aminolevulinic acid for resection of malignant glioma: a randomised controlled multicentre phase III trial, In The Lancet Oncology, 7(5),: 392-401. [PubMed: 16648043]

7. Paleologos TS, Wadley JP, Kitchen ND, Thomas DGT, Chandler WF (2000) Clinical utility and cost-effectiveness of interactive image-guided craniotomy: clinical comparison between conventional and image-guided meningioma surgery, Neurosurgery, Vol.47 no.1, 4048

8. Schulz C, Waldeck S, Mauer UM (2012) Intraoperative image guidance in neurosurgery: development, current indications, and future trends, Radiology Research and Practice, Vol.2012, 19

9. Bayer S, Maier A, Ostermeier M, Fahrig R, (2017) Intraoperative imageing modalities amd compensation for brain shift in tumor resection surgery, Int J Biomed Imag, Vol.2017, Article ID: 6028645

10. Gerard IJ, Kersten-Oertel M, Petrecca K, Sirhan D, Hall JA (2017) Brain shift in neuronavigation of brain tumors: A review, Med Image Anal, Vol.35, 403-420 [PubMed: 27585837] 
11. Hata N, Nabavi A, Warfield S, Wells W, Kikinis R, Jolesz FA (1999) A volumetric optical flow method for measurement of brain deformation from intraoperative magnetic resonance images, MICCAI, LNCS Vol.1679, 928935, Taylor C and Colchester A, Eds.

12. Riva M, Hennersperger C, Milletari F, Katouzian A, Pessina F, Gutierrez-Becker B, Castellano A, Navab N, Bello L (2017) 3D intra-operative ultrasound and MR image guidance: pursuing an ultrasound-based management of brainshift to enhance neuronavigation, Int J CARS, DOI 10.1007/s11548-017-1578-5

13. Reinertsen I, Lindseth F, Unsgaard G, Collins DL (2007) Clinical validation of vessel-based registration for correction of brain-shift, Med Image Anal, vol.11, no.6, 673684 [PubMed: 17681484]

14. Letteboer MM, Willems PW, Viergever MA, Niessen WJ (2003) Non-rigid registration of 3D ultrasound images of brain tumours acquired during neurosurgery, MICCAI 2003, LNCS vol.2879, 408415, Ellis RE and Peters TM, Eds.

15. Toews M, Wells W (2013) Efficient and robust model-to-image alignment using 3D scale-invariant features, Med Image Anal, Vol 17, 271-282 [PubMed: 23265799]

16. Cressie NAC, Statistics for spatial data, p900 Wiley, USA (1991)

17. Calder CA, Cressie N (2009) Kriging and variogram models, Int Encyc of Human Geo, Vol.2009, $49-55$

18. Matheron G (1963) Principles of geostatistics. Econ Geol Vol.58, 12461266

19. Kikinis R, Pieper SD, Vosburgh K (2014) 3D Slicer: a platform for subject- 24 specific image analysis, visualization, and clinical support. Intraoperative Imaging Image-Guided Therapy, Jolesz FA, Editor Vol.3(19), p277289

20. Haslett J, Brandley R, Craig P, Unwin A, Wills G (1991) Dynamic Graphics for Exploring Spatial Data With Applications to Locating Global and Local Anomalies, The American Statistician, Vol. 45, 234-242

21. Tukey JW, Exploratory data analysis, p444, Addison-Wesely (1977) 


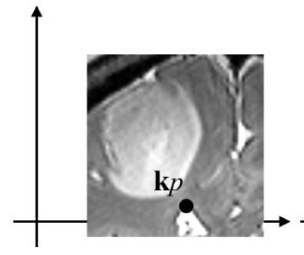

(a)

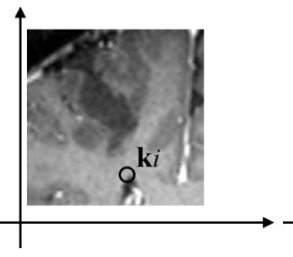

(b)

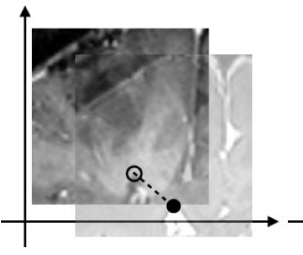

(c)

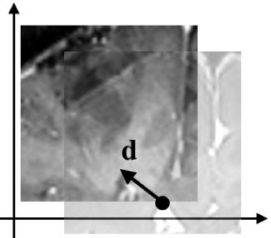

(d)

Fig. 1.

(a) The extracted key-point on the preoperative image; (b) The extracted key-point on the intraoperative image; (c) Overlaying two images; (d) The displacement vector $\mathbf{d}$ for the preoperative key-point. 


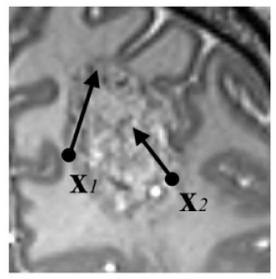

(a)

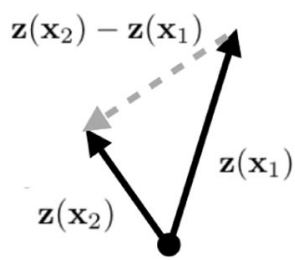

(b)

Fig. 2.

(a) Two key-points $\mathbf{x}_{1}, \mathbf{x}_{2}$; (b) The displacement differences between $\mathbf{z}\left(\mathbf{x}_{1}\right)$ and $\mathbf{z}\left(\mathbf{x}_{2}\right)$, and the lag $h$. 


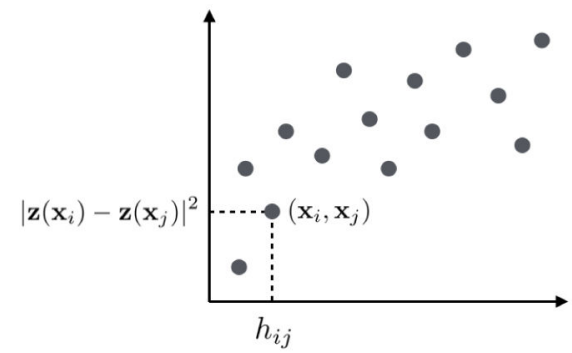

(a)

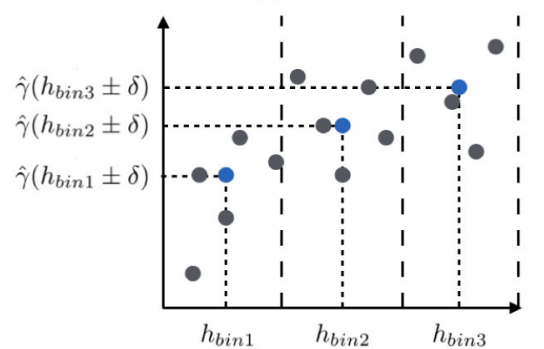

(c)

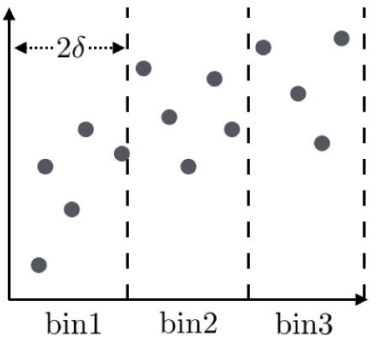

(b)

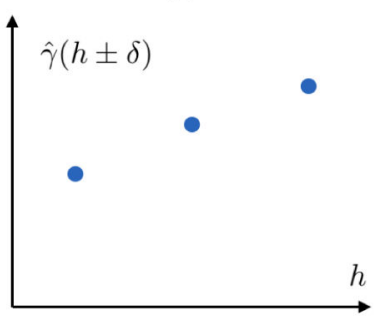

(d)

Fig. 3.

(a) The variogram cloud; (b) Binning the variogram cloud with the bin width $2 \delta$; (c) Computing the mean for every bin; (d)Plotting $\hat{\gamma}\left(h_{\text {bink }} \pm \delta\right)$ and $h_{\text {bink }}$. 


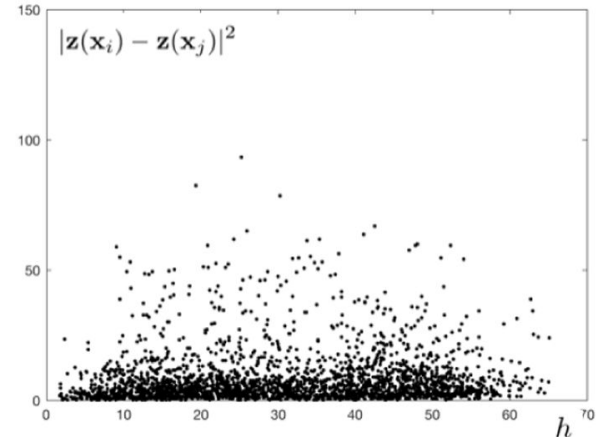

(a)

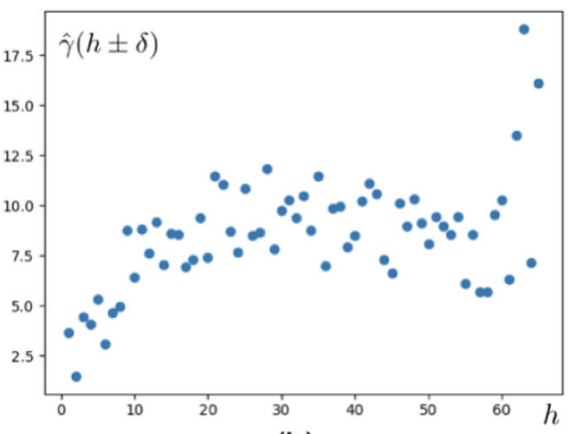

(b)

Fig. 4.

(a) The variogram cloud for a set of US data; (b)The empirical variogram for the same US data. 


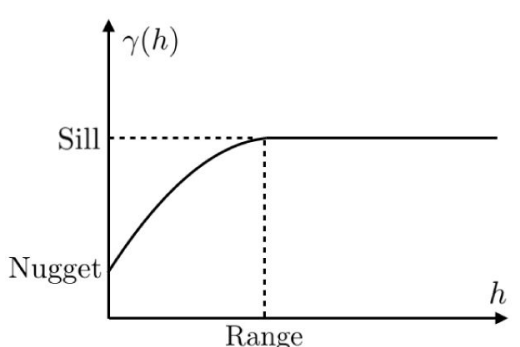

(a)

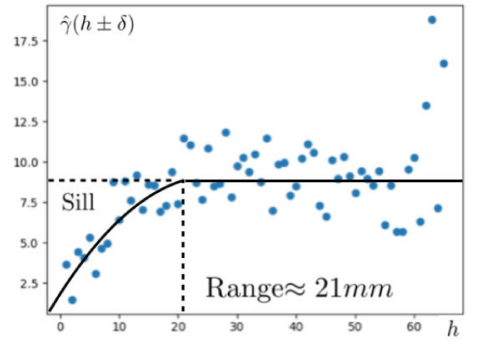

(b)

Fig. 5.

(a) Example of a fitted variogram model; (b) A continuous model fitted to the empirical variogram of the US data that has 71 key-point pairs. 

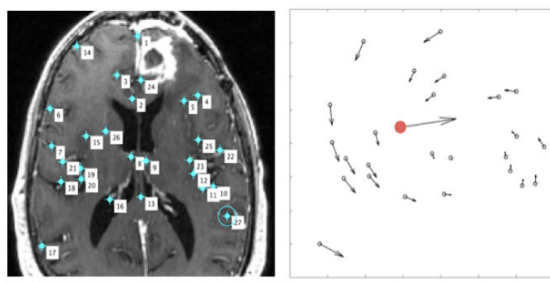

(a)

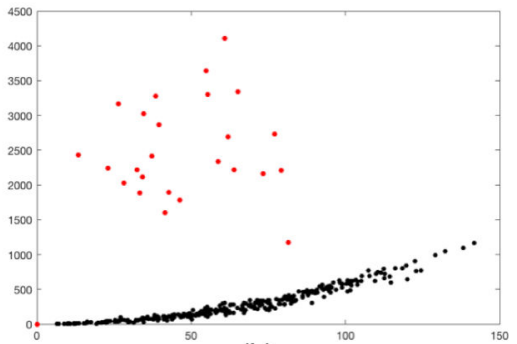

(b)

Fig. 6 .

(a) Displacement vectors and a red global outlier; (b) The global outlier identified in the variogram cloud. 

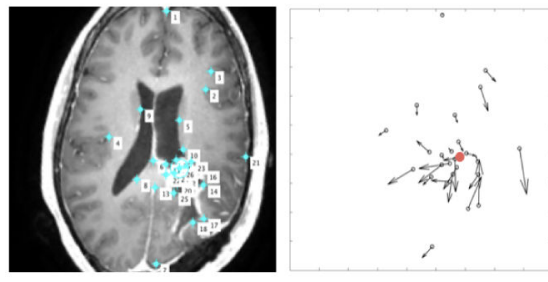

(a)

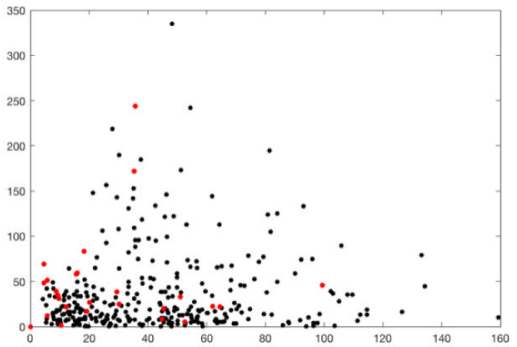

(b)

Fig. 7.

(a) Displacement vectors and a red local outlier; (b) The local outlier identified in the variogram cloud. 

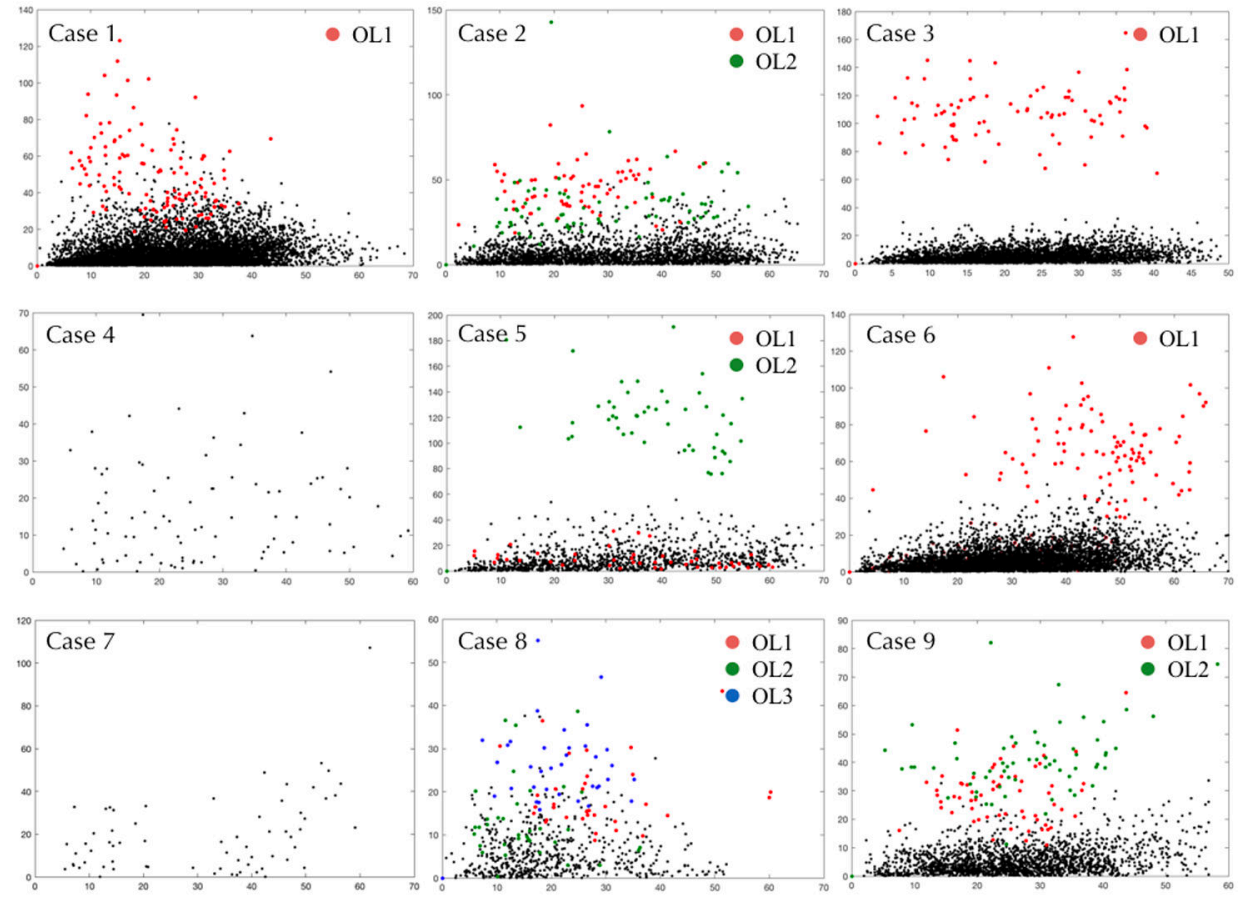

Fig. 8.

The variogram for all potential outliers that were flagged by the operator in a total of 9 cases. 

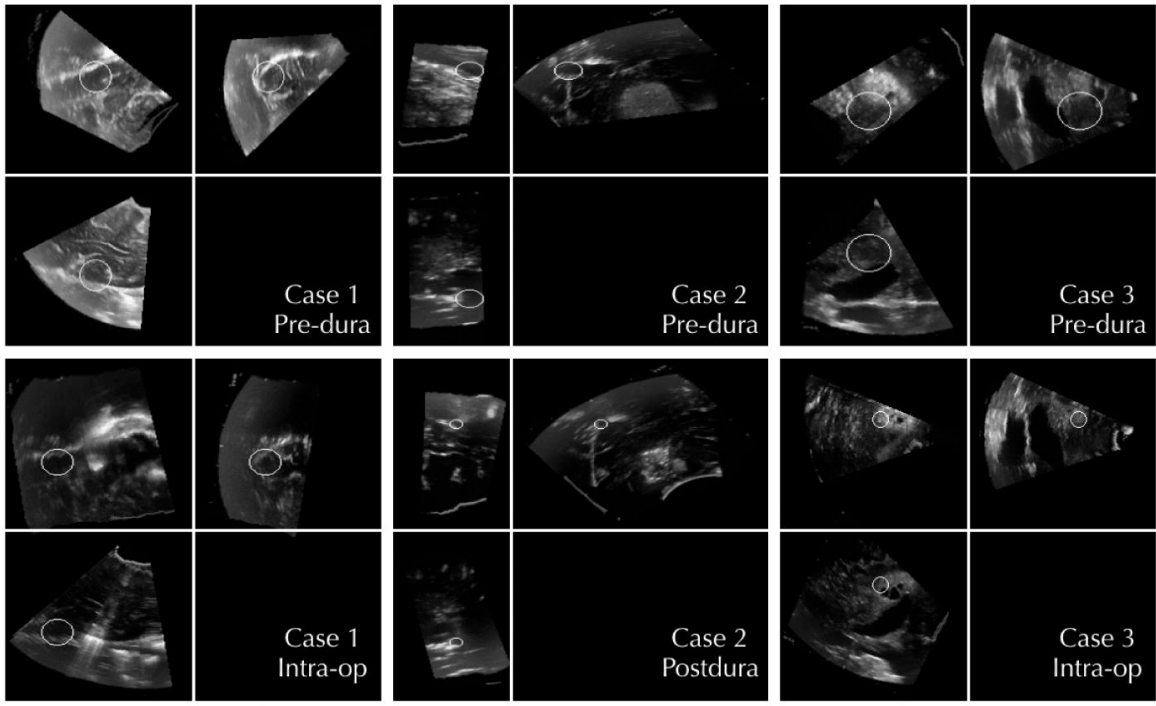

Fig. 9.

Screen shots of axial (top left), sagittal (bottom left) and coronal (top right) views of 3 outliers found by the variogram. Note the matching quality of the axial slice of case 1 , the sagittal slice of case 2 and the cases 3 are considerably bad. 


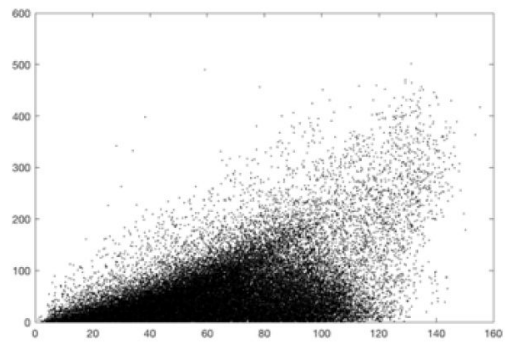

(a)

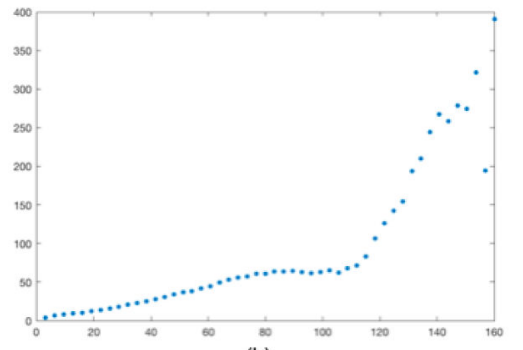

(b)

Fig. 10.

(a) The variogram cloud for the MRI data that has 386 matched key-point pairs; (b) The corresponding variogram. 
Table 1

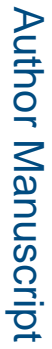

Experimental results of the proposed outlier screening method

\begin{tabular}{|c|c|c|c|c|c|c|l|l|l|}
\hline Case & $\mathbf{1}$ & $\mathbf{2}$ & $\mathbf{3}$ & $\mathbf{4}$ & $\mathbf{5}$ & $\mathbf{6}$ & $\mathbf{7}$ & $\mathbf{8}$ & $\mathbf{9}$ \\
\hline Data & intra & intra & postd & postd & intra & intra & intra & postd & intra \\
\hline Matches & 123 & 71 & 84 & 14 & 49 & 98 & 12 & 37 & 64 \\
\hline \#OL & 1 & 2 & 1 & 0 & 2 & 1 & 0 & 3 & 2 \\
\hline OLS1 & -1.3 & -1.4 & -2.3 & $\mathrm{n} / \mathrm{a}$ & -0.9 & -2.3 & $\mathrm{n} / \mathrm{a}$ & -0.8 & -2.0 \\
\hline OLS2 & $\mathrm{n} / \mathrm{a}$ & -1.9 & $\mathrm{n} / \mathrm{a}$ & $\mathrm{n} / \mathrm{a}$ & -2.2 & $\mathrm{n} / \mathrm{a}$ & $\mathrm{n} / \mathrm{a}$ & -0.4 & -2.2 \\
\hline OLS3 & $\mathrm{n} / \mathrm{a}$ & $\mathrm{n} / \mathrm{a}$ & $\mathrm{n} / \mathrm{a}$ & $\mathrm{n} / \mathrm{a}$ & $\mathrm{n} / \mathrm{a}$ & $\mathrm{n} / \mathrm{a}$ & $\mathrm{n} / \mathrm{a}$ & -1.8 & $\mathrm{n} / \mathrm{a}$ \\
\hline
\end{tabular}

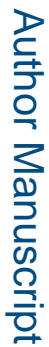

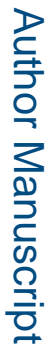

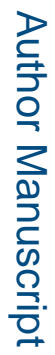

Int J Comput Assist Radiol Surg. Author manuscript; available in PMC 2019 December 01. 